\title{
Permeability Coefficient Inversion Method Based on Hybrid Algorithm
}

\author{
X. G. Liu ${ }^{1}$, G. H. Pei ${ }^{2, *}$ \\ ${ }^{1}$ Institute of Porous Flow and Fluid Mechanics, Chinese Academy of Sciences, Langfang 065007, China \\ ${ }^{2}$ Institute of Poromechanics, Wuhan Polytechnic University, Wuhan 430023, China \\ Email: peigh@whpu.edu.cn
}

\begin{abstract}
Precise numerical simulation to seepage zone is a significant part of water power project. The accuracy of numerical analysis to seepage zone depends on both the simulation extent of the numerical model and parameter determination. At the present, the mathematical seepage model is, to a large degree, perfect, but the parameter determining; especially the permeability coefficient is far from perfect. Indoor experiments measuring permeability coefficient is easily influenced by the sample uncertainty and scale effect. Permeability coefficient got from field test is relatively accurate, but the precision of result depend on the number and distribution of test points. By increasing the number of test points, the precision is improved. Correspondingly, the cost rises. It is a new manner to inverse the rock permeability coefficient through numerical model. The basic idea is set a numerical model of seepage field, by fitting the dynamic information (such as head, flux) of the movement of ground water to inverse the permeability coefficient of rock mass. Parameter got through inversion can reflect the seepage characteristics of the rock mass of target zone. But inversion methods exist two difficulties: result indeterminacy and heavy load of calculation.

Genetic algorithm developed from simulating life evolution system is a searching algorithm. Its applications in rock project and geophysics suggest that it can overcome indeterminacy of inverse problem. The biggest difficulty of genetic algorithm when used to parameter inversion is that its searching space is huge and the convergence speed is slow, which will result in too much calculation in the process of inversion. In order to overcome this defect, a mixed algorithm is established through the combination of genetic algorithm and optimistic method. As an improvement, this paper based on genetic algorithm is about permeability coefficient inversion, a global optimism of inversion result is assured. At the same time, in the process of inversion, some characteristics of seepage are considered, and a local searching process is added to establish a mixed algorithm to reduce searching space and increase convergence speed. The application of the algorithm in the permeability coefficient inversion of a gravity dam site indicates that the optimism of inversion result is efficiently assured and, hence, the uniqueness of solution, but also quicken convergence and reduce calculation burden.
\end{abstract}

\section{REFERENCES}

1. Chen G, Xu HL. Space scale effect of porous media permeability. Geology Scientific and Technical Information, 1998; 17(2): 27-34

2. Zhu YM, Zhang LJ. Inversion analysis of permeability tensor of fractured rock mass. Chinese Journal of Rock Mechanics and Engineering, 1997; 16(5): 461-470

3. Gao W, Zheng YR. Evolution inversion method of rockmass parameters. Chinese Journal of Hydraulic Engineering, 2000; 8: 1-5

4. Sambrige M. Genetic algorithm in seismic waveform inversion. Int. J. Geophy., 1992; 109: 323-342 